\title{
Prospects for a better diagnosis and prognosis of NAFLD: a pathologist's view
}

\author{
Carolin Lackner \\ Institute of Pathology, Medical University of Graz, Graz 8010, Austria.
}

Correspondence to: Dr. Carolin Lackner, Institute of Pathology, Medical University of Graz, Neue Stiftingtalstraße 6, Graz 8010, Austria. E-mail: karoline.lackner@medunigraz.at

How to cite this article: Lackner C. Prospects for a better diagnosis and prognosis of NAFLD: a pathologist's view. Hepatoma Res 2021;7:27. https://dx.doi.org/10.20517/2394-5079.2020.137

Received: 30 Oct 2020 First Decision: 24 Nov 2020 Revised: 4 Jan 2021 Accepted: 12 Jan 2021 Available online: 9 Apr 2021

Academic Editor: Stefano Bellentani Copy Editor: Xi-Jun Chen Production Editor: Yue-Yue Zhang

\begin{abstract}
Despite the development of surrogate non-invasive methods, histological evaluation remains an important tool for reliable classification, grading and staging, as well as prognosis in non-alcoholic fatty liver disease (NAFLD). However, histological evaluation has been criticised because it requires a liver biopsy, its propensity for sampling, and inter-observer variation. This article highlights the future developments in the morphological interpretation of liver biopsy in NAFLD, so as to aid in improving its diagnostic and prognostic utility.
\end{abstract}

Keywords: Non-alcoholic fatty liver disease, histology, prognosis, grading and staging

\section{CURRENT PROPOSALS FOR A CHANGE IN TERMINOLOGY OF FATTY LIVER DISEASES}

Traditionally, the liver manifestation of the metabolic syndrome has been known as non-alcoholic fatty liver disease (NAFLD). Recently, a group of experts proposed to change the name of this condition into metabolic-dysfunction-associated fatty liver disease (MAFLD) to highlight the underlying systemic metabolic dysfunction as the one of the primary causes of fatty liver disease $\mathrm{e}^{[1,2]}$. In addition, some experts also suggested that for patients with both MAFLD and other risk factors for fatty liver disease such as alcohol abuse, the term "dual-etiology fatty liver disease" should be used. Currently the validity and utility of the proposed new terminology is debated ${ }^{[3,4]}$. In the light of ongoing discussion and since neither of the terms, MAFLD or "dual-etiology fatty liver disease", has yet been endorsed by major liver societies the traditional term, NAFLD, is used in this review.

The Author(s) 2021. Open Access This article is licensed under a Creative Commons Attribution 4.0 International License (https://creativecommons.org/licenses/by/4.0/), which permits unrestricted use, sharing, adaptation, distribution and reproduction in any medium or format, for any purpose, even commercially, as long as you give appropriate credit to the original author(s) and the source, provide a link to the Creative Commons license, and indicate if changes were made. 


\section{EPIDEMIOLOGY OF NON-ALCOHOLIC FATTY LIVER DISEASE}

NAFLD is a major health concern. The ongoing epidemic of obesity provides the basis for the striking rise in incidence and prevalence of NAFLD around the world. Currently, the estimated global prevalence of NAFLD is $25 \%{ }^{[5]}$ and it is expected to increase substantially in the next decade: mortality and advanced liver disease due to NAFLD have been estimated to more than double during 2016-2030 ${ }^{[6]}$. Cirrhosis, end-stage liver disease, and/or hepatocellular carcinoma due to NAFLD are also the leading indications for liver transplantation. NAFLD-related morbidity will continue to negatively impact public health and contribute to the escalating health-care $\operatorname{cost}^{[7]}$.

\section{TYPES OF NAFLD}

NAFLD comprises a spectrum of diseases ranging from steatosis [non-alcoholic fatty liver (NAFL)] to nonalcoholic steatohepatitis (NASH) and NAFLD-associated fibrosis or cirrhosis. The types of NAFLD are defined by histology ${ }^{[8]}$. Morphological lesions of NAFLD are accentuated in centrilobular regions. NAFL refers to the accumulation of fat vesicles in hepatocytes (macrovesicular steatosis), affecting $\geq 5 \%$ of hepatocytes. In most cases, fatty change is associated with some degree of lobular inflammation. Inflammatory infiltrates are typically mild consisting mostly of mononuclear cells and eventually few admixed neutrophils. NASH is characterized by three key features: fatty change of hepatocytes, hepatocellular ballooning, and lobular inflammation.

Ballooning refers to a special form of hepatocellular degeneration that is characterized by enlargement and rounding of the cytoplasm contributed by the loss of the intermediate filament (IF) cytoskeleton, accumulation of small droplet fat, dilation of the ER as well as fluid retention. Ballooned hepatocytes often contain irregularly garland-shaped hyaline cytoplasmic inclusions, which consist of aggregated K8, K18, and a number of other stress-related proteins (reviewed $\mathrm{in}^{[0]}$ ).

Fatty change and inflammation- and ballooning-associated hepatocellular injury contribute to endoplasmatic reticulum (ER) stress and the activation of a tightly regulated cellular network consisting of macrophages, natural killer cells, B cells, and natural killer T cells, all of which control hepatic stellate cell activation. This results in the deposition of extracellular matrix containing collagen as well as other proteins around small groups and individual hepatocytes [reviewed in ${ }^{[10]}$, giving rise to the so-called perisinusoidal or pericellular fibrosis (PSF and PCF, respectively); for simplicity the latter term is used in this manuscript], which is typical for fatty liver diseases. PCF can progress and extend from centrilobular areas to involve other portions of hepatic lobules connecting central veins and portal tracts (bridging the fibrosis). Eventually, in an ongoing liver injury, dense fibrous septa develop and destroy the lobular architecture. Parenchymal nodules without portal-central relations surrounded by fibrous septa are hallmarks of cirrhosis. In NAFLD-associated cryptogenic cirrhosis ${ }^{[11]}$, the features of disease activity-steatosis, ballooning and inflammation-may subside and PCF too may no longer be a feature ${ }^{[12]}$.

\section{Prognostic relevance and challenges in the histological diagnosis of NASH}

The diagnosis of NASH is of clinical relevance ${ }^{[13]}$ since it helps to identify patients who are at risk for the development of cirrhosis, liver failure, hepatocellular carcinoma, cardiovascular disease, and extrahepatic malignancies, which eventually result in elevated liver-related as well as overall mortality ${ }^{[1,15]}$. The diagnosis of NASH requires a liver biopsy, an invasive procedure that is subject to a small, but not insignificant risk of morbidity and mortality. A plethora of non-invasive methods to diagnose NASH have been developed. However, presently, the diagnostic accuracy of these approaches is not optimal. Therefore, current clinical practice guidelines issued by international scientific societies recommend liver biopsy and histological evaluation for patients in whom a diagnosis of NAFLD is uncertain or NAFLD-related advanced liver 
disease is suspected ${ }^{[16]}$.

Hepatocellular ballooning is pivotal for the distinction between NAFL with relatively benign prognosis and NASH with considerably worse prognosis. It is also an integral feature of all current morphological grading and staging systems of NAFLD. Furthermore, clinical studies in NAFLD evaluating the utility of novel therapeutic strategies frequently include only those patients who have a histologically confirmed NASH.

Although the morphological features of NASH are well described, its histological diagnosis can be challenging. A great proportion of the diagnostic difficulties is related to the reliable recognition of ballooned hepatocytes in H\&E-stained sections. Ballooning refers to a spectrum of morphological changes in the hepatocellular cytoplasm and its contents (see above) rather than a single feature that is subject to a moderate inter-observer agreement in the morphological interpretation ${ }^{[17,18]}$. However, several immunohistochemical markers for ballooned hepatocytes have been described. Oxidative stress-related rearrangement of the IF cytoskeleton leads to a loss of K8- and K18-associated staining of the cytoplasm. In contrast to normal-sized hepatocytes, the cytoplasm of ballooned cells is negative with antibodies against K8 and K18; whereas Mallory-Denk bodies containing the aggregated forms of K8 and K18, are positive ${ }^{[19]}$. Ballooned hepatocytes have also been shown to express sonic hedgehog promoting pathways responsible for fibrogenesis in hedgehog-responsive stroma cells in the vicinity of ballooned hepatocytes and the development of $\mathrm{PCF}^{[20]}$ [Figure 1]. In addition, expression of aldose reductase, AKR1B10, presumably involved in the detoxification of oxidative stress-associated reactive aldehydes in NASH can also be detected by respective antibodies in ballooned hepatocytes ${ }^{[21]}$. These markers could be used to confirm ballooning by immunohistochemistry in doubtful cases and increase inter-observer agreement.

\section{PROGNOSTIC IMPLICATIONS OF FIBROSIS STAGES IN NAFLD}

Although NASH is associated with an increased risk for the development of fibrosis, several studies have identified fibrosis stages as the most important prognostic factor for NAFLD ${ }^{[22,23]}$. A large meta-analysis including 1495 NAFLD patients with approximately 17-year of follow-up showed that stage 1 NAFLD patients have an increased all-cause mortality risk, and this risk increases with progression to higher stages, whereas liver-related mortality increases exponentially after progress to stage 2 . These data indicate that all cause- and liver-related mortality is predicted by individual fibrosis stages ${ }^{[14]}$. Furthermore, individual fibrosis stages are associated with cause-specific mortality in NAFLD. In a multi-national study including 458 NAFLD patients, liver-related events were predominantly associated with the cirrhosis stage, whereas patients with the bridging fibrosis stage were more prone to develop non-hepatic cancers and vascular events $^{[15]}$. However, non-invasive fibrosis tests are not suited to accurately discriminate between individual histological fibrosis stages, thereby impacting accurate prognosis as well as efficient patient management.

Histology also offers information about the quality of fibrosis and has potential important implications in predicting the prognosis of NAFLD. Fibrogenesis is a complicated process involving hepatocytes, mesenchymal, and hematological cells, which leads to an excess accumulation and insufficient degradation of extracellular matrix (ECM) in the presence of a persistent liver injury (reviewed with emphasis on NAFLD in ${ }^{[24]}$ ). In addition, fibrogenesis can be propelled by platelets, endothelial, and stellate cells via liver injury-associated activation of the coagulation cascade and thrombin production (reviewed in ${ }^{[25]}$ ). However, if liver injury subsides, e.g., after antiviral therapy in patients with chronic viral hepatitis, degradation of ECM and hepatocellular regeneration leads to resorption of ECM and restitution of the lobular architecture. Fibrosis regression can occur in pre-cirrhotic as well as, to a certain extent, in the cirrhotic stage ${ }^{[26-28]}$. Histological features of fibrosis vary between progressive and regressive disease processes. Recently, the Beijing classification of liver fibrosis was developed (reviewed in $^{[29]}$ ) [Table 1]. This classification system 

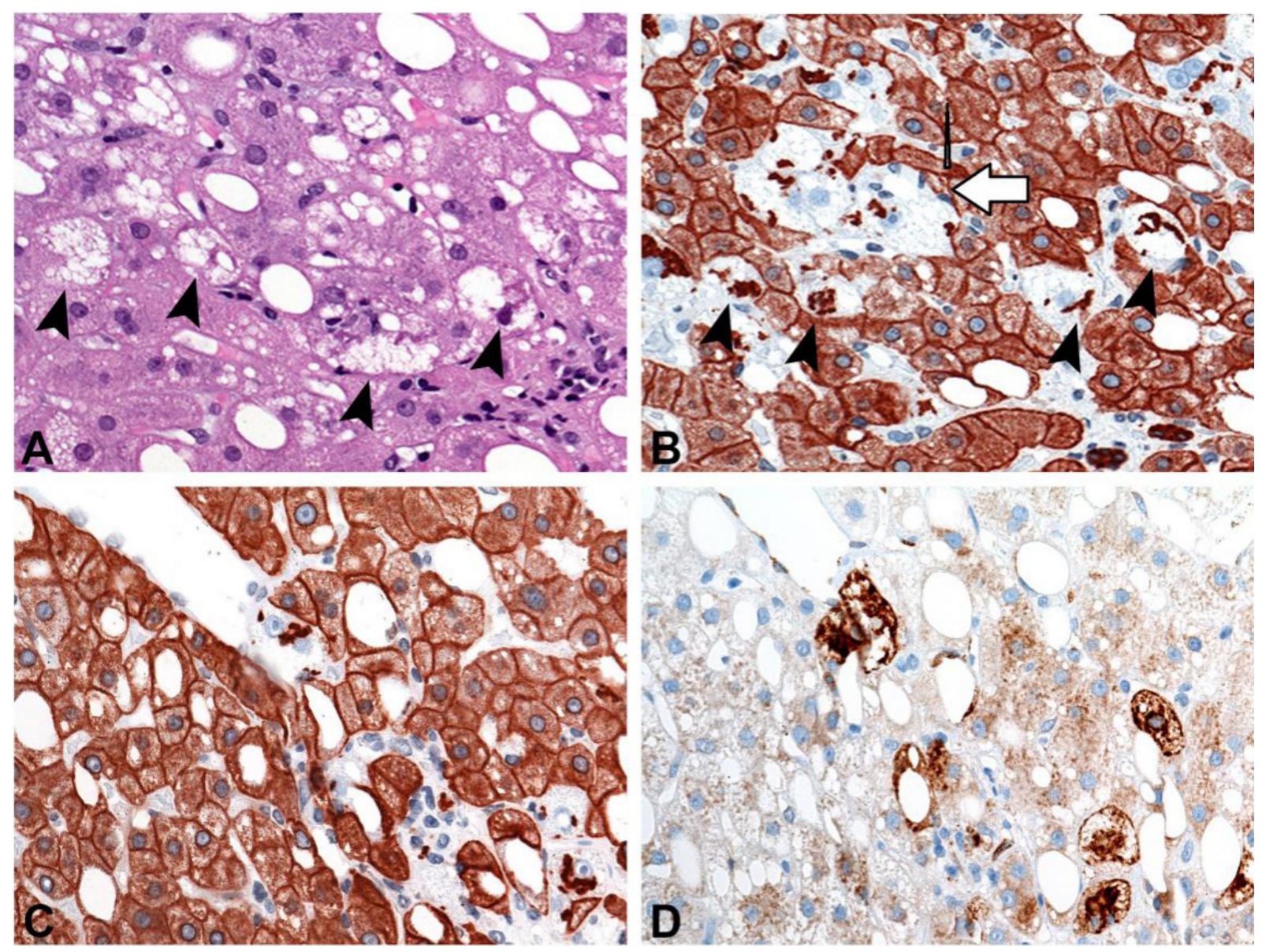

Figure 1. Immunohistochemical markers for the detection of hepatocellular ballooning (A-D). (A) Ballooned hepatocytes (indicated by arrow heads) with enlarged lightly stained cytoplasm containing lipid droplets and Mallory-Denk bodies (MDBs) are present among hepatocytes with fatty change. (B) Consecutive section of the HE stained section in (A). Immunohistochemical analysis reveals loss of cytoplasmic and positive MDB-staining signals with $\mathrm{K} 8 / 18$ antibodies in the ballooned hepatocytes (marked by arrow heads). In addition, in this deeper section another small group of ballooned hepatocytes with MDBs not clearly detectable in the respective $\mathrm{H \& E}$ stain is indicated by the characteristic K8/18 staining pattern (ballooned hepatocytes are indicated by white arrow heads). (C) Ballooned hepatocytes adjacent to a central vein with loss of cytoplasmic K8/18 staining and K8/18-positive MDBs are strongly marked in a consecutive section (D) with antibodies against sonic hedgehog whereas the cytoplasm of normal sized hepatocytes shows only weak reactivity.

provides a tool to not only stage fibrosis, but also to differentiate between progressive and regressive fibrosis types that are associated with outcomes. In contrast to patients with a fibrosis pattern that indicated regression, individuals with a progressive or indeterminate (balance of pro- and regressive features) fibrosis pattern continued to progress despite successful suppression of a viral infection ${ }^{[30]}$. Although the Beijing classification has been developed for the interpretation of biopsies of patients with viral hepatitis, it could also be applied in other chronic liver diseases like NAFLD ${ }^{[29]}$. However, studies specifically addressing this issue are currently lacking.

Another factor defining the quality of fibrosis is the composition of ECM. Data from animal as well as human studies suggest that paucicellular scar tissue present in dense fibrous septa that bridges fibrosis and cirrhosis consist of, among other proteins, cross-linked collagen and elastic fibres, which are more resistant to degradation than the ECM of early fibrosis stages and/or PCF. It is conceivable that the composition of ECM may thus differ with respect to a progression- or regression-related biological setting ${ }^{[31-34]}$. 
Unfortunately, data on the prognostic utility of ECM composition in patients with NAFLD are sparse.

Dual-photon microscopy-based quantitation of fibrosis-related parameters (q-FP) is an interesting novel method for the refinement of hepatic fibrosis assessment. The q-FP analysis measures the quantity of collagen and also provides information on the architectural features of collagen fibers. Quantitative collagen and the collagen fiber pattern are strongly correlated to the NASH Clinical Research Network (CRN) fibrosis stages and represent an accurate and reproducible way to characterize fibrosis along a continuous scale in NAFLD ${ }^{[35]}$.

\section{IMPACT OF STANDARDS FOR GRADING AND STAGING NAFLD}

Grading and staging refer to the semiquantitative assessment of inflammation and hepatocellular injury (activity) and fibrosis (stage) by the application of numerical scores. Grading and staging systems are used in many chronic liver diseases to predict a prognosis, guide patient management, assess treatment effects in clinical trials, and as research tools ${ }^{[36-38]}$. Several grading and staging systems have been described for NAFLD, among which the NAFLD activity score (NAS), the Clinical Research Network (CRN) staging system $^{[17]}$ and the steatosis, activity and fibrosis (SAF) scores ${ }^{[39]}$ are the most widely used. These are important tools assisting the diagnosis of NASH and have been validated for clinical practice ${ }^{[40,41]}$ [Table 2].

The natural course of NAFLD may be reflected in an improvement or worsening of morphological disease activity (indicated by the NAS and possibly also the SAF activity score), which is associated with regression or progression of fibrosis, respectively ${ }^{[42]}$. In clinical trials of NAFLD, the currently accepted endpoints for a conditional approval of pharmaceutical agents include resolution of NASH without worsening of the fibrosis and/or improvement in fibrosis without worsening of NASH, as assessed by a standardized histological evaluation of paired liver biopsies using the NAS and CRN staging systems ${ }^{[43]}$. Therefore, reliable recognition of key features of activity as well as stage are pivotal for the interpretation of results of clinical trials.

However, morphological interpretation is prone to inter-observer bias ${ }^{[44]}$. In the NAS and SAF systems, descriptive terms define the scores for morphological changes of grade and stage. To some extent, these definitions allow for a range of interpretations. For example, macrovesicular steatosis, a component of the NAS, is assessed according to the degree of "parenchymal involvement", which can be interpreted as the percentage of hepatocytes containing macrovesicular fat or the proportion of parenchymal area contributed by macrovesicular lipid droplets [Figure 2]. By the same token, an uncertainty with respect to the exact definitions of other morphological features of grade and stage exists. Therefore, scores will vary among individual observers depending on the definitions used, which impacts the diagnostic categories and therefore has obvious consequences in the clinical management as well as in the selection of patients for clinical trials. However, inter-observer variation can be markedly reduced by a settlement on morphological standards prior to the histological evaluation using tutorial guidelines, diagnostic algorithm $\mathrm{s}^{[39]}$, and guideline images ${ }^{[45]}$. In a study on the utility of the SAF score and the fatty liver inhibition of progression diagnostic algorithm ${ }^{[39]}$, the rate of agreement with a reference diagnosis increased after the application of the algorithm from $77 \%$ to $97 \%$ among expert liver pathologists, and from $42 \%$ to $75 \%$ among pathologists with a more general training. Similarly, the inter-observer agreement for the detection of hepatocellular ballooning and lobular inflammation, NASH features traditionally plagued by only fair to moderate kappa values $^{[17,18]}$, reached almost perfect and substantial levels $(\kappa=0.8$ and 0.72 , respectively) with tutorial guidance. The same was true for the interpretation of stage, for which almost perfect agreement $(\kappa=0.84)$ was found. 


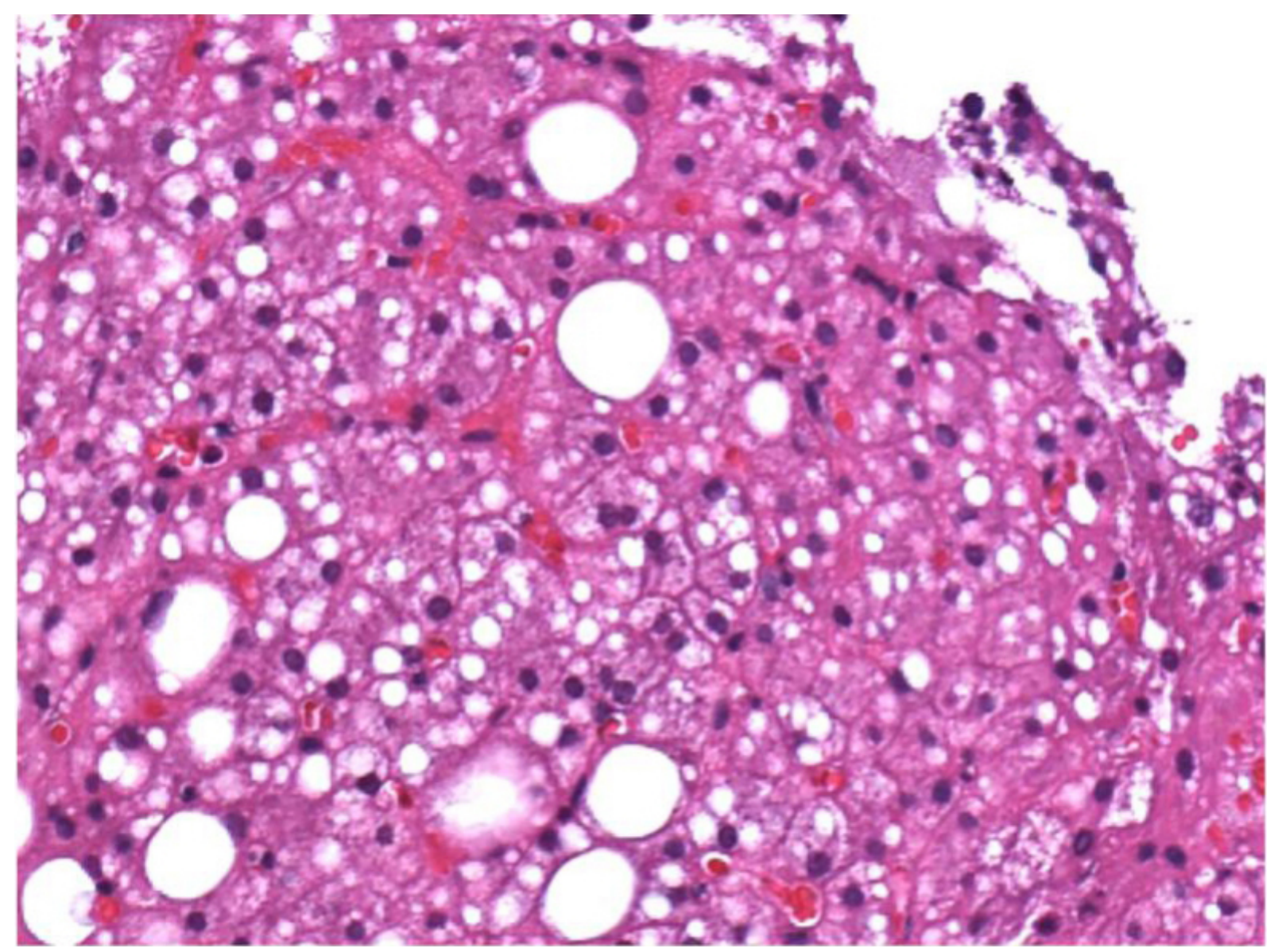

Figure 2. Impact of the morphological definition of steatosis on the results of semiquantitative grading. Approximately two thirds of the hepatocytes contain lipid droplets of variable sizes. However, if steatosis is defined by parenchymal areas contributed by lipid droplets the estimated degree of fatty change is considerably lower.

Recently, computer models have been developed to standardize the assessment of morphological features of grade and stage in NAFLD. Furthermore, semi-quantitative assessments can be replaced by quantitative measurements by using softwares that automatically evaluates steatosis, inflammation, ballooning, and fibrosis in liver tissue sections. It is expected that machine learning algorithms and quantification tools will improve reproducibility in the interpretation of grade and stage, which is required for clinical practice as well as for clinical trials of $\mathrm{NASH}^{[46,47]}$.

\section{SUMMARY}

The classical histological interpretation of liver biopsies is an important tool for the diagnosis, classification as well as prognosis of NAFLD. Currently, histology is required for the exact diagnosis of NASH, the identification of individual fibrosis stages associated with diverging prognostic and therapeutic scenarios, as well as for the semi-quantitative assessment of grading and staging NAFLD. Developments in novel morphological methods such as computer-assisted digital image analysis, dual-photon microscopy, and the use of immunochemistry hold great promise in overcoming limitations related to the diagnosis and the inter-observer variability of grading and staging NAFLD. Future studies evaluating the applicability and utility of these novel methods for clinical practice and clinical trials are warranted. 


\section{DECLARATIONS}

\section{Authors' contributions}

The author contributed solely to the article.

\section{Availability of data and materials}

Not applicable.

\section{Financial support and sponsorship}

None.

\section{Conflicts of interest}

The author declared that there are no conflicts of interest.

\section{Ethical approval and consent to participate}

Not applicable.

\section{Consent for publication}

Not applicable.

\section{Copyright}

(c) The Author(s) 2021.

\section{REFERENCES}

1. Eslam M, Newsome PN, Sarin SK, et al. A new definition for metabolic dysfunction-associated fatty liver disease: An international expert consensus statement. J Hepatol 2020;73:202-9. DOI PubMed

2. Eslam M, Sanyal AJ, George J, International Consensus Panel. MAFLD: a consensus-driven proposed nomenclature for metabolic associated fatty liver disease. Gastroenterology 2020;158:1999-2014. DOI PubMed

3. Younossi ZM, Rinella ME, Sanyal A, et al. From NAFLD to MAFLD: implications of a premature change in terminology. Hepatology 2020. DOI PubMed

4. Moreno C, Sheron N, Tiniakos D, Lackner C, Mathurin P; EASL Consortium for the Study of Alcohol-related LiVer disease in Europe (SALVE). "Dual aetiology fatty liver disease": a recently proposed term associated with potential pitfalls. J Hepatol 2020:S01688278(20)33755. DOI PubMed

5. Younossi ZM, Koenig AB, Abdelatif D, Fazel Y, Henry L, Wymer M. Global epidemiology of nonalcoholic fatty liver disease-Metaanalytic assessment of prevalence, incidence, and outcomes. Hepatology 2016;64:73-84. DOI PubMed

6. Estes C, Anstee QM, Arias-Loste MT, et al. Modeling NAFLD disease burden in China, France, Germany, Italy, Japan, Spain, United Kingdom, and United States for the period 2016-2030. J Hepatol 2018;69:896-904. DOI PubMed

7. Cotter TG, Rinella M. Nonalcoholic fatty liver disease 2020: the state of the disease. Gastroenterology 2020;158:1851-64. DOI PubMed

8. Brunt EM. Nonalcoholic steatohepatitis: pathologic features and differential diagnosis. Semin Diagn Pathol 2005;22:330-8. DOI PubMed

9. Lackner C. Hepatocellular ballooning in nonalcoholic steatohepatitis: the pathologist's perspective. Expert Rev Gastroenterol Hepatol 2011;5:223-31. DOI PubMed

10. Schwabe RF, Tabas I, Pajvani UB. Mechanisms of Fibrosis Development in Nonalcoholic Steatohepatitis. Gastroenterology 2020;158:1913-28. DOI PubMed PMC

11. Younossi Z, Stepanova M, Sanyal AJ, et al. The conundrum of cryptogenic cirrhosis: Adverse outcomes without treatment options. $J$ Hepatol 2018;69:1365-70. DOI PubMed

12. Caldwell SH, Crespo DM. The spectrum expanded: cryptogenic cirrhosis and the natural history of non-alcoholic fatty liver disease. $J$ Hepatol 2004;40:578-84. DOI PubMed

13. Association for the Study of the Liver (EASL), European Association for the Study of Diabetes (EASD), European Association for the Study of Obesity (EASO). EASL-EASD-EASO Clinical Practice Guidelines for the management of non-alcoholic fatty liver disease. $J$ Hepatol 2016;64:1388-402. DOI PubMed

14. Dulai PS, Singh S, Patel J, et al. Increased risk of mortality by fibrosis stage in nonalcoholic fatty liver disease: systematic review and meta-analysis. Hepatology 2017;65:1557-65. DOI PubMed PMC

15. Vilar-Gomez E, Calzadilla-Bertot L, Wai-Sun Wong V, et al. Fibrosis severity as a determinant of cause-specific mortality in patients with advanced nonalcoholic fatty liver disease: a multi-national cohort study. Gastroenterology 2018;155:443-57.e17. DOI PubMed

16. Leoni S, Tovoli F, Napoli L, Serio I, Ferri S, Bolondi L. Current guidelines for the management of non-alcoholic fatty liver disease: a 
systematic review with comparative analysis. World J Gastroenterol 2018;24:3361-73. DOI PubMed PMC

17. Kleiner DE, Brunt EM, Van Natta M, et al; Nonalcoholic Steatohepatitis Clinical Research Network. Design and validation of a histological scoring system for nonalcoholic fatty liver disease. Hepatology 2005;41:1313-21. DOI PubMed

18. Younossi ZM, Gramlich T, Liu YC, et al. Nonalcoholic fatty liver disease: assessment of variability in pathologic interpretations. Mod Pathol 1998;11:560-5. PubMed

19. Lackner C, Gogg-Kamerer M, Zatloukal K, Stumptner C, Brunt EM, Denk H. Ballooned hepatocytes in steatohepatitis: the value of keratin immunohistochemistry for diagnosis. J Hepatol 2008;48:821-8. DOI PubMed

20. Rangwala F, Guy CD, Lu J, et al. Increased production of sonic hedgehog by ballooned hepatocytes. J Pathol 2011;224:401-10. DOI PubMed PMC

21. Starmann J, Fälth M, Spindelböck W, et al. Gene expression profiling unravels cancer-related hepatic molecular signatures in steatohepatitis but not in steatosis. PLoS One 2012;7:e46584. DOI PubMed PMC

22. Ekstedt M, Hagström H, Nasr P, et al. Fibrosis stage is the strongest predictor for disease-specific mortality in NAFLD after up to 33 years of follow-up. Hepatology 2015;61:1547-54. DOI PubMed

23. Taylor RS, Taylor RJ, Bayliss S, et al. Association between fibrosis stage and outcomes of patients with nonalcoholic fatty liver disease: a systematic review and meta-analysis. Gastroenterology 2020;158:1611-25.e12. DOI PubMed

24. Schwabe RF, Tabas I, Pajvani UB. Mechanisms of fibrosis development in nonalcoholic steatohepatitis. Gastroenterology 2020;158:1913-28. DOI PubMed PMC

25. Bitto N, Liguori E, La Mura V. Coagulation, microenvironment and liver fibrosis. Cells 2018;7:85. DOI PubMed PMC

26. Jung YK, Yim HJ. Reversal of liver cirrhosis: current evidence and expectations. Korean J Intern Med 2017;32:213-28. DOI PubMed PMC

27. Ellis EL, Mann DA. Clinical evidence for the regression of liver fibrosis. J Hepatol 2012;56:1171-80. DOI PubMed

28. Marcellin P, Gane E, Buti M, et al. Regression of cirrhosis during treatment with tenofovir disoproxil fumarate for chronic hepatitis B: a 5-year open-label follow-up study. Lancet 2013; 381:468-75. DOI PubMed

29. Theise ND, Jia J, Sun Y, Wee A, You H. Progression and regression of fibrosis in viral hepatitis in the treatment era: the Beijing classification. Mod Pathol 2018;31:1191-200. DOI PubMed

30. Sun Y, Zhou J, Wang L, et al. New classification of liver biopsy assessment for fibrosis in chronic hepatitis B patients before and after treatment. Hepatology 2017;65:1438-50. DOI PubMed

31. Pellicoro A, Aucott RL, Ramachandran P, et al. Elastin accumulation is regulated at the level of degradation by macrophage metalloelastase (MMP-12) during experimental liver fibrosis. Hepatology 2012;55:1965-75. DOI PubMed

32. Pellicoro A, Ramachandran P, Iredale JP. Reversibility of liver fibrosis. Fibrogenesis Tissue Repair 2012;5:S26. DOI PubMed PMC

33. Pellicoro A, Ramachandran P, Iredale JP, Fallowfield JA. Liver fibrosis and repair: immune regulation of wound healing in a solid organ. Nat Rev Immunol 2014;14:181-94. DOI PubMed

34. Issa R, Zhou X, Constandinou CM, et al. Spontaneous recovery from micronodular cirrhosis: evidence for incomplete resolution associated with matrix cross-linking. Gastroenterology 2004;126:1795-808. DOI PubMed

35. Wang Y, Vincent R, Yang J, et al. Dual-photon microscopy-based quantitation of fibrosis-related parameters (q-FP) to model disease progression in steatohepatitis. Hepatology 2017;65:1891-903. DOI PubMed PMC

36. Ishak K, Baptista A, Bianchi L, et al. Histological grading and staging of chronic hepatitis. J Hepatol 1995;22:696-9. DOI PubMed

37. Bedossa P, Poynard T. An algorithm for the grading of activity in chronic hepatitis C. The METAVIR Cooperative Study Group. Hepatology 1996;24:289-93. DOI PubMed

38. Wendum D, Boëlle PY, Bedossa P, et al. Primary biliary cirrhosis: proposal for a new simple histological scoring system. Liver Int 2015;35:652-9. DOI PubMed

39. Bedossa P, FLIP Pathology Consortium. Utility and appropriateness of the fatty liver inhibition of progression (FLIP) algorithm and steatosis, activity, and fibrosis (SAF) score in the evaluation of biopsies of nonalcoholic fatty liver disease. Hepatology 2014;60:56575. DOI PubMed

40. Nascimbeni F, Bedossa P, Fedchuk L, et al. Clinical validation of the FLIP algorithm and the SAF score in patients with non-alcoholic fatty liver disease. J Hepatol 2020;72:828-38. DOI PubMed

41. Nascimbeni F, Ballestri S, Machado MV, et al. Clinical relevance of liver histopathology and different histological classifications of NASH in adults. Expert Rev Gastroenterol Hepatol 2018;12:351-67. DOI PubMed

42. Kleiner DE, Brunt EM, Wilson LA, et al. Association of histologic disease activity with progression of nonalcoholic fatty liver disease. JAMA Netw Open 2019;2:e1912565. DOI PubMed PMC

43. Rinella ME, Tacke F, Sanyal AJ, Anstee QM; participants of the AASLD/EASL Workshop. Report on the AASLD/EASL joint workshop on clinical trial endpoints in NAFLD. J Hepatol 2019;71:823-33. DOI PubMed

44. Davison BA, Harrison SA, Cotter G, et al. Suboptimal reliability of liver biopsy evaluation has implications for randomized clinical trials. J Hepatol 2020;73:1322-32. DOI PubMed

45. Hall AR, Green AC, Luong TV, Burroughs AK, Wyatt J, Dhillon AP. The use of guideline images to improve histological estimation of hepatic steatosis. Liver Int 2014;34:1414-27. DOI PubMed

46. Liu F, Goh GB, Tiniakos D, et al. qFIBS: an automated technique for quantitative evaluation of fibrosis, inflammation, ballooning, and steatosis in patients with nonalcoholic steatohepatitis. Hepatolog;71:1953-66. DOI PubMed

47. Garcia-Carretero R, Vigil-Medina L, Barquero-Perez O, Ramos-Lopez J. Relevant features in nonalcoholic steatohepatitis determined using machine learning for feature selection. Metab Syndr Relat Disord 2019;17:444-51. DOI PubMed 